\title{
The integrated Earth system model version 1: formulation and functionality
}

\author{
W. D. Collins ${ }^{1}$, A. P. Craig ${ }^{2}$, J. E. Truesdale ${ }^{2}$, A. V. Di Vittorio ${ }^{3}$, A. D. Jones ${ }^{3}$, B. Bond-Lamberty ${ }^{4}$, K. V. Calvin ${ }^{4}$,

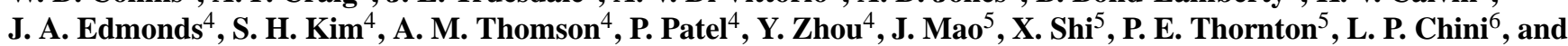 \\ G. C. Hurtt $^{6}$ \\ ${ }^{1}$ University of California, Berkeley and Lawrence Berkeley National Laboratory, Berkeley, CA, USA \\ ${ }^{2}$ Independent contractors with Lawrence Berkeley National Laboratory, Berkeley, CA, USA \\ ${ }^{3}$ Lawrence Berkeley National Laboratory, Berkeley, CA, USA \\ ${ }^{4}$ Joint Global Change Research Institute, College Park, MD, USA \\ ${ }^{5}$ Oak Ridge National Laboratory, Oak Ridge, TN, USA \\ ${ }^{6}$ University of Maryland, College Park, MD, USA
}

Correspondence to: W. D. Collins (wdcollins@lbl.gov, wdcollins@ berkeley.edu)

Received: 26 October 2014 - Published in Geosci. Model Dev. Discuss.: 21 January 2015

Revised: 21 May 2015 - Accepted: 27 May 2015 - Published: 23 July 2015

\begin{abstract}
The integrated Earth system model (iESM) has been developed as a new tool for projecting the joint human/climate system. The iESM is based upon coupling an integrated assessment model (IAM) and an Earth system model (ESM) into a common modeling infrastructure. IAMs are the primary tool for describing the human-Earth system, including the sources of global greenhouse gases (GHGs) and short-lived species (SLS), land use and land cover change (LULCC), and other resource-related drivers of anthropogenic climate change. ESMs are the primary scientific tools for examining the physical, chemical, and biogeochemical impacts of human-induced changes to the climate system. The iESM project integrates the economic and humandimension modeling of an IAM and a fully coupled ESM within a single simulation system while maintaining the separability of each model if needed. Both IAM and ESM codes are developed and used by large communities and have been extensively applied in recent national and international climate assessments. By introducing heretofore-omitted feedbacks between natural and societal drivers, we can improve scientific understanding of the human-Earth system dynamics. Potential applications include studies of the interactions and feedbacks leading to the timing, scale, and geographic distribution of emissions trajectories and other human influences, corresponding climate effects, and the subsequent impacts of a changing climate on human and natural systems.
\end{abstract}

This paper describes the formulation, requirements, implementation, testing, and resulting functionality of the first version of the iESM released to the global climate community.

\section{Introduction}

As documented extensively in the Intergovernmental Panel on Climate Change (IPCC) Fifth Assessment Report (AR5) (IPCC, 2014), there is now broad scientific consensus that not only has the climate of the 20th and early 21 st centuries changed from its recent historical baseline, but also that those changes are in large part due to human actions and decisions. At the same time, there is now broad scientific understanding that it is highly likely that climatic change and the consequences that have already occurred will grow in both rate and magnitude during the 21 st century and present significant challenges to environmental quality, sustainable development, and the state and condition of both natural resources and human infrastructure (CCSP, 2008; GCRP, 2009).

Integrated assessment models (IAMs) are the primary tools for describing the human components of the Earth system, the sources of greenhouse gases (GHGs) and short-lived species (SLS) emissions, and drivers of land use change. Earth system models (ESMs) are the primary tools for examining the climatic, biogeophysical, and biogeochemical im- 
pacts of changes to the radiative properties of the Earth's atmosphere. These two modeling paradigms developed largely independently of each other and their interactions have historically been relatively simplistic. Typically, projections of GHGs and SLS emissions have been produced by the human system components of IAMs, archived in databases, and used by ESMs to produce projections of climate and altered biogeophysical processes.

As IAMs have become more sophisticated, they have gradually expanded to incorporate agriculture, land cover and land use change, and representations of the terrestrial carbon cycle because processes in those sectors affect anthropogenic emissions of GHGs and SLS in important and unavoidable ways. Many studies (e.g., van Vuuren et al., 2007; Clarke et al., 2007, 2009) have shown that limiting or stabilizing GHGs produces very different distributions of energy sources, energy use, and the use of land and other resources. ESMs have also evolved in the direction of endogenously including the natural processes in these same sectors, but have generally omitted the representations of the human components that drive changes in them and the resulting changes in emissions. Such changes due to human activity have either been specified a priori or have generally been taken directly from output of independent IAMs. Experiments of this sort have demonstrated the importance of land cover changes on simulations of the climate system (e.g., Pielke et al., 2002; Matthews et al., 2004; Snyder et al., 2004; Feddema et al., 2005; Fung et al., 2005; Pitman et al., 2009; Brovkin et al., 2013).

In conjunction with the World Climate Research Program (WCRP) Fifth Climate Model Intercomparison Program (CMIP5) and the IPCC AR5, these two modeling communities have engaged in an unprecedented degree of collaboration to ensure that the products of the IAM community meet the needs of the climate and Earth system modeling communities (Moss et al., 2010; Taylor et al., 2011; van Vuuren et al., 2011). Among the many CMIP5 experiments are those that use the output of IAMs in a one-way transfer of information of either emissions or concentrations of GHGs (as well as land use and land use change areas) to produce scenarios whose radiative forcing and direction of change are prescribed for the year 2100. Four Representative Concentration Pathway (RCP) scenarios with increasing levels of radiative forcing in 2100 were selected, namely, 2.6, 4.5, 6.0, and $8.5 \mathrm{~W} \mathrm{~m}^{-2}$ (van Vuuren et al., 2011). Each scenario was produced by a different IAM using different assumptions about land use change through the 21 st century. The research design envisioned the development of a literature that included the development of representative concentration pathways (RCPs) by many IAM teams using alternative underlying socioeconomic assumptions. This variety in turn would enable IAM researchers to explore uncertainty in the socioeconomic system driving emissions because, it was argued, any underlying socioeconomic system that produced a given radiative forcing pathway could be paired with the as-

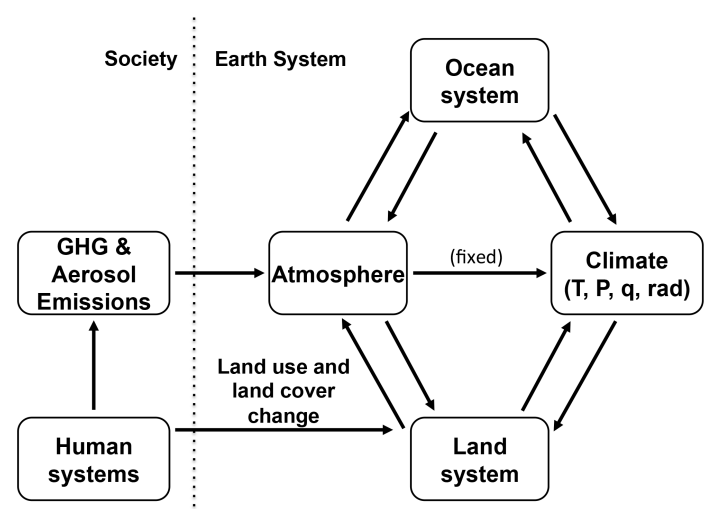

Figure 1. Illustration of the one-way coupling from the human to the climate system used in prior simulations of global environmental change.

sociated climate scenarios from the CMIP5 database (Moss et al., 2010; van Vuuren et al., 2011).

But as sophisticated as this interaction has become, it is still a one-way transfer of information from IAMs to ESMs (Fig. 1). It does not allow IAMs to easily examine the climate system consequences of changes in human decisions as represented in emissions pathways, nor does it allow the changing climate system to affect the human components of energy, water resources, or land use in a systematic fashion. Finally, it does not allow for an evaluation of how differences in human decision-making might affect either climate outcomes or the actual impacts of a changing physical climate system. However, the emerging observable impacts of climate-change mean that we can no longer assume that human energy and land systems that produce emissions are evolving under a static climate.

It is therefore clear that future work must enable the processes in these sectors to interact with each other and the climate system rather than to remain as one-way transfers of information. If ESMs are to include better representations of the feedbacks of climate change on agriculture, land use, land cover, and terrestrial carbon cycle, as well as other human systems such as energy and the economy, then they will need the ability to incorporate the human system directly. Heretofore the tools have not existed for a fully consistent representation of the combined evolution of these two systems.

In order to advance beyond this paradigm, we have developed a new model framework, the integrated Earth system model (iESM). The goal is to create a first-generation integrated system to improve climate simulations and enhance scientific understanding of climate impacts on human systems and important feedbacks from human activities to the climate system. The first version of the iESM described in this paper is designed to address three major science questions: (1) is the present CMIP5 "parallel process" approach to climate assessment adequate? (2) Will human activities affect local and regional climate on scales that matter? (3) Will cli- 


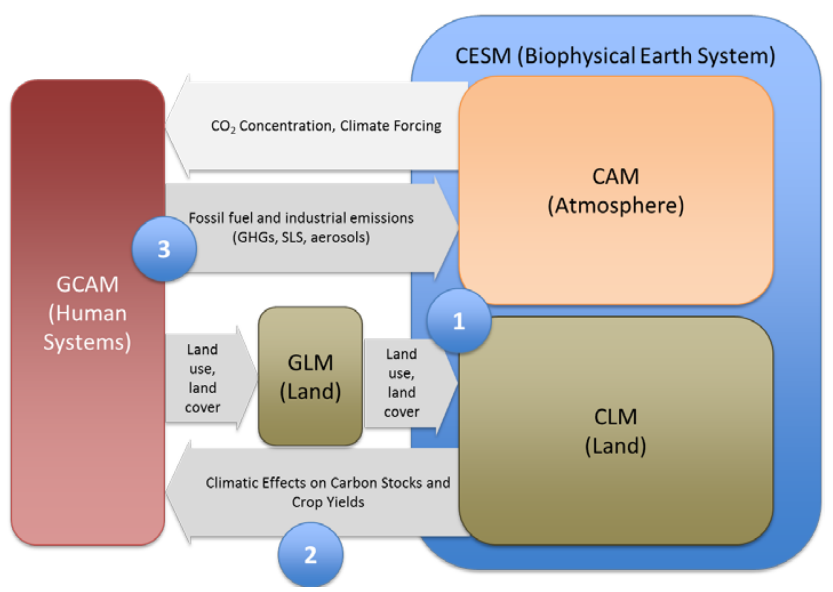

Figure 2. Schematic of the integrated Earth system model (iESM) showing its major component models GCAM, CESM, and GLM as well as the two-way connections between these models.

mate change itself affect human decision-making and its implications for biogeochemical and biogeophysical processes at global scales?

The iESM is a new configuration of models previously operated separately. The iESM includes the human system components of an IAM called the global change assessment model (GCAM) (Kim et al., 2006; Calvin, 2011; Wise et al., 2014), the complete community Earth system model (CESM) (Hurrell et al., 2013), and the global land use model (GLM) (Hurtt et al., 2011) for rendering GCAM output onto the spatial grid and transforming land use information for use by the Community Land Model (CLM) component of CESM (Lawrence et al., 2011; Oleson et al., 2013) (Fig. 2). GCAM and CESM are both community codes, and the resulting iESM is also being released to the global climate community.

The iESM includes both one-way and two-way communication of fluxes and feedbacks among the components of the energy and land use systems from GCAM, as well as the incorporation of their physical consequences for both biogeochemical and physical fluxes in CESM. This allows for the investigation of the degree to which this linkage may change the evolution of the climate system over decades to a century. We have used the iESM to investigate the climate impacts on human systems and important feedbacks from human activities to the climate system. The iESM results on impacts and feedbacks are described in a series of earlier and companion papers (e.g., Jones et al., 2012, 2013).

This paper describes the scientific rationale for the construction of the iESM (Sect. 2), the component models assembled to create it (Sect. 3), the requirements on the assembly process (Sect. 4), the technical implementation of the model (Sect. 5), and the procedures used to validate the linkages among the component models and ensure the integrity of the coupled system (Sect. 6). The paper concludes with fu- ture plans for further extensions and applications of the iESM (Sect. 7).

\section{Climate-change impact on energy demand, supply, and production}

Climate change can influence energy demand, supply, and production in several major areas. Energy demand for adaptation and mitigation measures may also increase under climate change (van Vuuren et al., 2012). Integrated assessment (IA) models can be used to explore consequences and responses of energy systems to climate change. In the IA modeling community, however, energy supply and demand are normally modeled based on historical conditions, and climate-change impacts are rarely incorporated except in a static manner. Although some efforts have begun to explore climate-change impacts on the energy system using IA models (Voldoire et al., 2007), only one-way coupling is usually employed, and the interactions between the energy system and climate are seldom addressed. Two-way coupling between human and Earth systems would be required to examine the impacts of climate change on (1) building energy use, (2) renewable energy potential, and (3) energy production (thermal power plants) and transmission, each of which is described in greater detail below.

\subsection{Building energy use}

Climate change can have important impacts on building energy systems through decreased heating and increased cooling. Previous studies are limited in addressing the effect of a changing climate on building energy demands while simultaneously considering other energy sectors in the underlying human systems. In recent years, the impacts of climate change on building energy use have been evaluated using IA models by constructing estimates of heating and cooling degree days from air temperature outputted from climate models (Isaac and van Vuuren, 2009; van Ruijven et al., 2011; Zhou et al., 2013, 2014; Yu et al., 2014). The feedback from the climate on the energy system was calculated from climate model output in advance using a one-way coupling scheme, and the impact of these changes in the energy system on climate was rarely considered in these studies. One exception is the study by Labriet et al. (2013), in which the climate change and building energy use was fully coupled with IA and climate models. However, the spatial resolution of climate outputs from this coupled modeling system was low $\left(5^{\circ}\right)$, and it may limit the understanding of climate-change impact on building energy use.

\subsection{Renewable energy production}

Renewable energy plays an important role in the energy system at the regional and global levels, and it can be influenced by climate change to a large extent. In current IA modeling 
efforts, the availability of renewable energy (i.e., wind, solar energy, and hydropower) and its economic potential are either modeled according to the historical condition (Zhang et al., 2010; Zhou et al., 2012) or exogenously quantified using proxies such as precipitation or runoff (Golombek et al., 2012). However, renewable energy resources, such as wind and hydropower, are dependent on the local climate that can be very different from current or historical conditions under climate change. For example, previous studies found that both wind speed and variability show changing trends in the historical time period (Holt and Wang, 2012; Zhou and Smith, 2013) that can impact wind energy potential. Climate change can also alter future photovoltaic and concentrated solar power energy output through changes in temperature and solar insolation (Crook et al., 2011). Hydropower potential can be influenced by precipitation and runoff changes under climate change, and previous studies found changes in hydropower potential under climate change globally and regionally (Hamududu and Killingtveit, 2012; de Lucena et al., 2009). Interactions between climate change and bioenergy are more complex because of changes in variables such as land use, which will in turn alter surface albedo and feedback on the climate (Schaeffer et al., 2006). The change of future renewable energy under climate change is rarely captured in current IA models, and the subsequent feedback of energy system change on climate systems has not been explored.

\subsection{Energy production}

Climate change also has important impacts on energy production, especially thermal power plants, which are influenced by the temperature of water used for cooling (Ruebbelke and Voegele, 2013, 2011) and which might also face limits to water availability in some cases. Increasing air and water temperature under climate change can reduce the efficiency of power plants. For example, it was found in a previous study that a $1{ }^{\circ} \mathrm{C}$ increase in temperature can reduce the supply of nuclear power by about $0.5 \%$ (Linnerud et al., 2011). In some extreme cases such as droughts and heat waves, power plants may not be able to meet temporary demand and may even shut down. Moreover, climate change such as extreme weather events and higher temperature also influence transmission lines through disruption of infrastructure or reduction of efficiency. The impact of climate change on thermal power plants was normally evaluated without consideration of the changes in other sectors in energy systems in previous studies (van Vliet et al., 2012; Foerster and Lilliestam, 2010; Ruebbelke and Voegele, 2013). Therefore, these studies of climate-change impact on energy production are necessarily limited without a more comprehensive understanding of the human system. IA models provide the possibility to evaluate the climate change on energy production in a comprehensive way. For example, a simple assumption has been made to evaluate the climate-change impact on thermal efficiency of power plants in the study by
Golombek et al. (2012), although there was still no feedback of change in the energy system back to the climate in this study.

\section{Models}

\subsection{The community Earth system model}

The starting point for the team's development efforts was version 1.0 (now 1.1) of the CESM. CESM is a community code and may be downloaded from the Community Earth System Model Project (2014; URL in references).

The CESM uses a flexible coupler that couples the atmosphere, ocean, land, and ice component models. Components often use different grids, and the coupler performs the necessary interpolation of fluxes and state variables. The CESM system comprises the Parallel Ocean Program, version 2 (POP), the CLM, version 4.0 (CLM 4.0), the Los Alamos sea ice model (CICE), the Community Atmosphere Model, version 5 (CAM), and the Community Ice Sheet Model (CISM). POP and CICE are finite volume codes with semi-implicit and explicit time integration and are implemented on logically Cartesian meshes that are stretched to embed polar singularities in land regions and thereby remove these singularities from computation.

The CAM model has flexible formulations for atmospheric dynamics, and it has recently transitioned to the spectral finite element method coupled to an extensive suite of subgrid physical parameterizations in its standard configuration. CAM runs on unstructured quadrilateral grids. The CLM contains a suite of column process parameterizations running at each grid point with no communication between grid points. CLM 4.0 represents surface and subsurface water, energy, carbon, and nitrogen dynamics with a nested hierarchical sub-grid treatment that allows glaciers, lakes, urban areas, agricultural fields, forest, grassland, and shrubland to share space on each grid cell. Incident radiation is intercepted in a two-layer canopy, with vegetation, soil, snow aging, and black carbon impacts on albedo. Subsurface processes include vertically resolved biogeochemistry, options for carbon and nutrient cycle parameterization, and recently improved treatment of wetlands and permafrost dynamics. CISM is based upon the Glimmer model, an open-source (GNU Public License, GPL), three-dimensional, thermomechanical ice sheet model designed to be interfaced to a range of global climate models.

In the fully coupled configuration, the CICE and POP component models run with a nominal displaced-pole grid spacing of $1^{\circ}$ (approximately $110 \mathrm{~km}$ at the Equator and $30 \mathrm{~km}$ in polar regions) and, for POP, 42 levels in the vertical. The CAM and CLM models run with a grid of with $0.9^{\circ} \times 1.25^{\circ}$ resolution with 30 and 10 vertical levels, respectively. CLM also includes a separate vegetation layer. The output of the CESM consists of monthly means of several 
hundred quantities, plus daily averages of a subset of these quantities and hourly output of some key variables.

\section{Development of land use and land cover change representation in CLM4}

A mechanistic representation for the influence of land use and land cover change (LULCC) on carbon, nitrogen, water, and energy cycles was developed and implemented for the CMIP5 land use harmonization (Oleson et al., 2010; Lawrence et al., 2012). This approach is designed to operate on the land use data stream provided by the GLM code after translation from the four basic land cover types of GLM into the 18 plant functional types (PFTs) of CLM. The CLM LULCC approach recognizes net annual losses and gains of vegetated area for each PFT within each grid cell. Net loss is treated as a reduction in PFT area with biomass densities kept constant; net gain is treated as an increment in PFT area with the introduction of very low initial carbon density on the new area. For PFTs with existing area on a given grid cell, net gains in area extend the existing area and expand the existing biomass to cover the new area. This dynamic LULCC in CLM 4 is one of several anthropogenic forcing factors influencing global biogeochemical cycles and surface energy balance and has been extensively evaluated (Shi et al., 2011, 2013; Mao et al., 2012a, b, 2013).

\subsection{The global change assessment model}

GCAM is a dynamic-recursive model with technology-rich representations of the economy, energy sector, land use, and water linked to a reduced-form climate model that can be used to explore climate-change mitigation policies, including carbon taxes, carbon trading, regulations and accelerated deployment of energy technology (Edmonds and Reilly, 1985; Kim et al., 2006; Calvin, 2011; Wise et al., 2014). GCAM is a community code and may be downloaded from the Joint Global Change Research Institute (2014; URL in references).

Regional population and labor productivity growth assumptions drive the energy and land use systems employing numerous technology options to produce, transform, and provide energy services as well as to produce agriculture and forest products and to determine land use and land cover. The GCAM model takes population, gross domestic product (GDP), technology efficiencies and costs, and certain policies as external boundary conditions and determines regional energy, land use, and emissions distributions as a result. GCAM, like all IAMs, is calibrated to a base year (e.g., 2005) to reflect differences in resource endowments, technology history, and consumer tastes across regions.

Using a run period extending from 1990 to 2100 at 5-year intervals, GCAM has been used to explore the potential role of emerging energy supply technologies and the GHG consequences of specific policy measures or energy technology adoption including $\mathrm{CO}_{2}$ capture and storage, bioenergy, hy- drogen systems, nuclear energy, renewable energy technology, and energy use technology in buildings, industry and the transportation sectors (e.g., Clarke et al., 2007, 2009).

GCAM is a Representative Concentration Pathway (RCP)class model. This means it can produce the emissions and land use outputs necessary to force a full Atmosphere-Ocean General Circulation Model (AOGCM) or ESM as in the CMIP5 process (Thomson et al., 2011). Output includes projections of future energy supply and demand and the resulting GHG emissions, radiative forcing, and climate effects of 16 GHGs, aerosols, and SLS at $0.5^{\circ} \times 0.5^{\circ}$ resolution, contingent on assumptions about future population, economy, technology, and climate mitigation policy.

For iESM, the time step of GCAM was reduced from 15year to a 5-year standard with flexible time step capability. This capability is important for scale consistency and compatibility with CESM code. In addition, the land component, which simulates supply of land products (food, energy, fiber), was completely reformulated to follow functional forms that define productivity as a function of geographic location, climatic conditions, and inputs, and thus made more consistent with physical earth system parameters (Wise and Calvin, 2011). A higher spatial resolution data set was compiled to allow for land productivity simulation in 151 global regions (Kyle et al., 2011). Finally, the post-processing code to downscale human emissions of $\mathrm{CO}_{2}$ from the GCAM 14region scale to a CAM-compatible grid was redeveloped and ported to the CESM by the iESM development team. The downscaling of short-term forcers is currently under development.

\subsection{The global land use model}

The GLM is a tool for computing annual, gridded, fractional land use states and all underlying land use transitions, including the age, area and biomass of secondary (recovering) lands and the spatial patterns of wood harvest and shifting cultivation, in a format designed for inclusion in Earth system models (Hurtt et al., 2006). GLM computes these land use patterns using an accounting-based method that tracks the fractions of cropland, pasture, urban area, primary vegetation, and secondary vegetation in each grid cell as a function of the land surface at the previous time step. The solution of the model is constrained with inputs and data including historical reconstructions and future projections of land use (e.g., crop, pasture, and urban applications), wood harvest, and potential biomass and recovery rate. GLM is publicly available and may be downloaded from the University of Maryland Global Ecology Lab (2014; URL in references).

GLM was selected as the primary tool to provide harmonized land use data sets (Hurtt et al., 2011; Brovkin et al., 2013) for the CMIP5 experiments (Taylor et al., 2011) as part of the IPCC AR5 (IPCC, 2014). For this project GLM was used to compute the land use states and transitions annually, for the years $1500-2100$, using data from IAMs for the 
years 1850-2100. GLM provided a continuous time series of land use data at half-degree spatial resolution in a format that could be used by a variety of ESMs consistent with both the historical data and future data from IAMs utilizing data from a variety of IAMs. Further information on this application of GLM is available from the Land-use Harmonization Project (2014; URL in references).

For use in iESM, GLM was modified to use GCAM data on a 5-year time step and to accept data partitioned by GCAM's 151 agri-ecological zones (AEZs) instead of GCAM's 14 socioeconomic regions. In addition, GLM was altered to use the forest area data from GCAM and to spatially rearrange agricultural area within each AEZ to match potential forest area changes from GCAM.

\section{Requirements for the coupling among GCAM, GLM, and CESM1}

To ensure that the iESM is reliable, flexible, and extensible, its technical implementation follows from an extensive set of requirements that are detailed below.

\subsection{Implementation of iESM as an extension of CESM}

The primary goal of the development is to implement the iESM as an extension of the CESM to include a humandimension component. This requirement implies that the IAM is treated as a new component in CESM and the protocols applied to the five existing components are adopted for the human component as well. To conform with these protocols, the human-dimension component has been integrated into CESM's software environment, including CESM's configure and build procedures, execution protocols, input and output conventions, and regression testing procedures. The execution protocols include CESM's procedures for synchronizing the coupling and time stepping of its various components and for exchange of fields among these components that conform with the conservation laws (e.g., conservation of mass) governing the dynamic evolution of the whole system.

The developers have also ensured that the iESM conforms to CESM's standards for repeatable experiments, including exact restarts and use of machine-independent representations for the initial, boundary, and restart data sets. CESM has adopted the Network Common Data Format (NetCDF) for these data sets to utilize its features for representation of numerical fields that can be transparently exchanged across computational platforms. This is complemented by the requirement that iESM conform to CESM's standards for hardware and software portability. This requirement helps ensure that experiments with iESM are, in principle, strictly repeatable assuming that the underlying software and hardware configuration has been validated by the CESM project. In practice, a precise description of the boundary and ini- tial conditions, together with a detailed description of the model and its functionality, are needed to attain experimental reproducibility. To address this need, it follows that the functionality of the human-dimensions component should be clearly and comprehensively documented. The documentation should encompass individual pieces such as GCAM, GLM, the land use translator (LUT) code, as well as the pre/post-processing code which operates on the data exchanged within the human-dimensions component.

\subsection{Flexible modes of execution}

The second principal goal is to incorporate and extend CESM's flexible modes of execution to iESM. The flexibility has two main dimensions: first, the trade-off between the physical completeness and complexity of the model and its execution speed; second, the equivalence between two-way communication between components with the introduction of feedbacks through their joint interaction. The first type of flexibility is realized by incorporating several versions of each critical component that range from very simple to very complete representations of the component dynamics, with a corresponding range from inexpensive to intensive computational resource demands. The second type of flexibility is implemented by introducing versions of each component that either produce the same output state (e.g., a climatology read from data file) regardless of the input state, or that compute an output state based on the input state combined with its evolution equations. The omission or inclusion of two-way communication corresponds to the omission or inclusion of feedbacks between the given component and the rest of the model system.

Both types of flexibility are realized by incorporating three basic versions of each component known as "stub", "data", and "active" versions. The "stub" version is used primarily for automated testing of the system integration and performs some very rudimentary housekeeping functions in response to commands from the integration layer of the whole CESM. The "data" version produces a time-evolving state through spatial and/or temporal interpolation applied to a fixed timedependent input read from data files. The same state is reproduced regardless of the evolution and dynamics of the remainder of the coupled system. This version is computationally inexpensive but does not include the two-way feedbacks between the given component and the rest of the system present in the real world. The "active" version produces a time-evolving state governed by its initial conditions, a representation of the fundamental dynamical equations that pertain to that component, and the boundary conditions supplied by the rest of the coupled model system. This version is computationally intensive but includes the two-way feedbacks present in the real world.

To conform with this protocol, the iESM includes the stub and data version of the human-dimensions component, as well as the fully interactive assessment model GCAM. The 
stub and data versions are automatically tested to ensure that they are integrated and operating correctly using the same general test procedures applied to the existing components of CESM.

\subsection{Bilateral exchange among components of the coupled system}

CESM utilizes a set of standard protocols to implement bilateral exchange among components of the coupled system, and these protocols have been adopted for internal communications within the human component as well as including GCAM, GLM, the LUT code that prepares GLM output for input into CLM, and the associated interfaces. These protocols ensure that the modes of interaction and exchange among components are visible, reproducible, flexible, and extensible.

The visibility follows from the requirement that all fields are exchanged through a single, top-level, standardized communication mechanism. This mechanism is capable of recording all information input to and output from all model components, together with the operations performed by the coupling layer to enable the exchanges. This capability also ensures that the interactions are strictly reproducible, since all exchanges are managed and recorded by one standardized communication mechanism.

This mechanism can be configured at run time to add arbitrary numbers of fields to be exchanged among any given pair of components. This ensures that the communication protocol can support increasingly complete and complex interactions among components using the same well-tested framework, without the need for lengthy modifications to the underlying software.

iESM has adopted these conventions for exchanging information to integrate the functional parts within the humandimensions component and, ultimately, to couple the humandimensions component to other components in CESM. In the first implementation, the data passed between the humandimensions components and the rest of CESM are exchanged using data files to minimize the modifications to the existing CESM components. However, these data exchanges can be readily upgraded to the standard top-level interfaces, internal memory, and message passage adopted by the rest of CESM.

This solution automatically includes provisions for exchanging additional data, in particular the exchange of more or all of the forcing agents covered by the RCP handshake protocol (tntcat.iiasa.ac.at:8787/RcpDb/). The information exchanged at the interfaces between the human component and the rest of CESM depend on the precise experimental configuration. However, the interfaces themselves are invariant under changes in configuration to guarantee that a single set of communication software can be thoroughly and repeatedly tested and validated.

\subsection{Methodologies to treat the ranges in spatial and temporal resolution across iESM}

The IAM solves for the evolution of the human system using a fundamental assumption of market quasi-equilibrium, namely, that the inputs and outputs into energy generation, food production, and land resources are balanced on sufficiently large spatial and temporal scales. The length and timescales required for the market equilibrium assumption to hold are orders of magnitude larger than the corresponding scales used to solve the equations of motion for physical, chemical, and biogeochemical processes in the Earth system.

This disparity introduces a requirement on the design of the iESM, namely, to implement a flexible and extensible mechanism to handle differences in spatial and temporal resolutions between the human and physical components. To meet this requirement, iESM should include capabilities in temporal interpolation or accumulation (e.g., time averaging, or other operations) in all the interfaces depend on the ratio of time steps between the transmitting and receiving components linked by the interface. Similarly, spatial interpolation or accumulation should be included with the recognition that some preprocessing may be needed to prepare input data files to manage regridding.

These capabilities are consolidated into the interfaces among the human component and the rest of the CESM system to avoid "hard wiring" any assumptions about gradations in resolutions into the components themselves. The efficient exchange of data across different spatial grids is highly contingent on efficient communication between components and within a single component on highly distributed and massively parallel supercomputers. The interfaces are therefore based upon a common foundation of communication infrastructure that has been optimized to maximize computational throughput. In turn, the exchange of data between components operating on very different time steps introduces a strong dependency on the time management procedures for the whole coupled system. This dependency has been satisfied by completely prescribing the sequence of component execution, the interlaced calls to the interfaces, and the interpolation-accumulation operations in each interface call.

While CESM is designed for hybrid execution in any combination of serial and/or parallel execution of its various components, in the initial version of iESM the human component is run in serial mode. This mode of operation is necessitated by the multi-year time step of GCAM. Since the version GCAM used in iESM runs as a single-threaded application while the rest of the CESM is inherently multithreaded, the processor elements devoted to the non-human components are idle while GCAM is run for a single time step. This introduces the risk that iESM utilizes computational resources much less efficiently than the parent CESM. It is therefore necessary to evaluate the relative cost of the human-dimensions component to ensure it is not a performance or memory bottleneck and re-factor or parallelize 
code as needed. Fortunately, the overall CESM performance is only marginally impacted by the introduction of this serial code.

\subsection{Dual use capability and single code repository for GCAM}

GCAM and GLM, like the other components of CESM, are research codes and are therefore under continual development and extension by their primary developers and by the wider GCAM and land use communities. Recent developments include significant new capabilities directly relevant to studies of human-Earth system interactions, for example the introduction of supply and demand for water resources (Hejazi et al., 2013, 2014). In order to ensure that the humandimension capabilities of iESM stay current with IA science, the iESM developers have chosen to enhance GCAM and GLM so that these models could both run in their standard stand-alone modes or as parts of the iESM. Once these enhancements are incorporated in the main GCAM and GLM repositories, GCAM and GLM have dual-use capabilities as stand-alone models or elements of iESM, and these capabilities would be easily propagated to future versions with new scientific features of interest to both the GCAM and iESM communities. These future versions can then be extracted from the respective repositories to easily update iESM.

This design introduces several subsidiary requirements for the input to and output from GCAM and GLM. First, GCAM's and GLM's native input and output procedures must be extended as needed to perform file I/O in stand-alone mode to exchange data that are compatible with CESM. This in turn requires introducing input and output interfaces into GCAM and GLM that generalize the methods for information exchange to include message passing. As a result, the results from GCAM and GLM are indistinguishable whether using files or inline communication techniques to exchange data with the rest of iESM.

\subsection{A simplified and robust run-time environment for the GLM component}

One of the challenges in constructing iESM is the complexity of the historical land use and land cover data required for the downscaling operations performed by GLM. In order to create a much simpler and more robust run-time environment for the GLM component, several important modifications are necessary. These include collating and converting the numerous input and output data sets into a much small number of NetCDF files. It was also helpful to standardize GLM's control interface to provide a simple and robust way to manage GLM functionality. To reduce the considerable demands for memory from GLM, it was necessary to re-factor GLM's data and control structures as needed to reduce its large in-memory footprint. Because CESM must meet a re- quirement for exact (bit-for-bit) restarts, it was necessary to extend GLM's functionality to add a restart capability.

\subsection{Reproduction of the offline-coupled implementation of iESM}

To the extent feasible, it would be advantageous to have the coupled iESM reproduce the offline-coupled implementation using separate models. To meet this requirement, it is necessary to construct tests ensuring that the data exported by each interface agrees with the corresponding information exchanges in the offline-coupled implementation to the precision of the stand-alone implementation. In turn, these tests are based upon and therefore require a core level of state output and diagnostics to allow iESM to be validated against relevant observations and documented CESM/GCAM/GLM control runs.

\section{Implementation of the coupling among GCAM, GLM, and CESM}

The first phase of iESM code development was designed to update and codify the experimental protocol from CMIP5 to incorporate land use change and emissions of GHGs and SLS from GCAM into CESM, such that the models exchanged information at each time step rather than as a single, fullcentury pass at the start of the model future period (2005). The software development team acquired the GCAM and GLM model codes and incorporated them into the land node of CESM through a new component, the integrated assessment component (IAC). The IAC is currently visible only to the land model when run in iESM mode and drives prognostic land use change. Because the functionality of GCAMGLM is encapsulated within a CESM component, it can also be replaced by a data model, enabling testing with a range of IAMs.

Code modifications were made to GCAM such that the model looks to CESM for instruction on when to begin each new time step. Thus, the first version of the coupled model operates by GCAM projecting land use, then CESM projecting climate and ecosystem change and returning productivity information to GCAM, which then incorporates that information into the land use decisions for the next time step.

The code has been tested and is running on leadershipclass computing facilities at ORNL (Oak Ridge National Laboratory) (the Titan Cray XK7) and NERSC (National Energy Research Supercomputing Center) (the Hopper Cray XE6 and Edison Cray XC30) and has also been tested and configured on the DOE IARP (Integrated Assessment Research Program) cluster at PNNL/UMD (Pacific Northwest National Laboratory/University of Maryland) (Evergreen). The iESM code has also kept pace with current CESM versions, and was most recently updated (in summer 2013) to 
run with CESM 1.1.2, the most recent CESM release with a full carbon cycle spin-up available.

Scientific challenges were encountered in the design of the coupling between the CESM and the IAC component, specifically with regard to faithfully representing CESM's land productivity passed into the IAC as well as capturing the land use change being returned. These challenges were identified and solved through a series of soft-coupled runs where the project team ran each model one time step forward at a time and passed model output between them, as well as a series of offline, CLM-only runs with the IAC enabled. In this fashion, the coupling steps were refined while the software development was under way. This first development phase focused on the land use change components of the models. In parallel, algorithms to downscale GCAM GHG and SLS emissions have been developed and tested, and the code has been transferred to the development team.

\subsection{General IAC implementation}

The IAC is implemented like a standard component of CESM. The IAC component has stub, data, and active versions called SIAC, DIAC, and GIAC, respectively, that provide a range of functionalities and capabilities for the IAC component. The active IAC version (GIAC) is fully prognostic and runs the full suite of IAC subcomponents to produce dynamically varying land use/change data using carbon feedback scalars from CLM. The data component (DIAC) replaces the active GCAM/GLM sub-models with data derived from an offline IAM/GLM control run. It is currently used for testing and model spin-up, but in principle it could be used to force CESM with an arbitrary scenario, for example one of the three CMIP5 RCPs generated by an IA model other than GCAM. The stub component (SIAC) serves the same purpose as a CESM stub model, namely, to serve as a placeholder to satisfy interface requirements when the active or data component is not being run. The stub IAC is the default mode for CLM, which makes it $100 \%$ bit-for-bit backward compatible with the current CLM.

Like other CESM components, IAC has routines to initialize its state, execute by evolving forward in time, and complete its operations by communicating its new state and generating history and restart (check-point) files. While these routines do not satisfy all aspects of the current CESM interface standard, they could be readily modified to do so in the future. The checkpoint/restart mechanism built for the IAC meets the CESM requirements for exact restarts to facilitate long integrations of the model system. Following the template of other CESM components, the IAC has a built-in clock, a top-level interface that mimics a CESM component, a centralized collection of control information implemented via a standard Fortran namelist, and a set of clock, grid, control and field parameters defined in a shared module for query by and exchange with other parts of the model system. All the coupling within the IAC is done via internal memory.
While the IAC was initially implemented as a separate component in CESM, we have placed the IAC component beneath the land model, since the all the coupling in the initial version of iESM would involve the CESM land component. Because we are using a mature coupling strategy, we can easily reposition the IAC component as needed in the future. But for the moment, the IAC is implemented as an option in CLM, and therefore the IAC model resides in its own subdirectory within the main code base for CLM. The stub, active, or data mode of IAC is set via the CESM configuration files. When this mode is set to stub, the results from iESM are identical at the bit-for-bit level with the corresponding version of the conventional CESM. All the input data sets and namelist parameters for the IAC are set by enhanced versions of the namelist generation procedures for CLM.

In the current iESM, the IAC is built as part of the compilation of the CLM code. The procedure that builds CLM calls scripts that build the IAC model. The IAC compilation is done for the stub, data, or active version of the IAC model depending on the mode specified by the user. Most of the IAC code is written in Fortran 90 or C, and leverages the CESM makefile. When the active IAC model is specified, GCAM is built via GCAM's build scripts that have been modified slightly to support coupling while retaining support for GCAM's implementation in C++. Currently, coupling between the IAC and CLM components is done via data files to leverage current CLM capabilities and to minimize changes to CLM. The IAC reads data from CLM history files at the start of a time step and writes data to a time-varying surface data set at the end of the IAC time step. Both sets of data evolve in time as the coupled system advances.

\subsection{IAC design}

The IAC component consists of five subcomponents, including the models GCAM and GLM and the interfaces IAC2GCAM, GCAM2GLM, and GLM2IAC between these models and the rest of the IAC component (Fig. 3). The sequence in which these subcomponents are invoked starts with IAC2GCAM, proceeds through GCAM, GCAM2GLM, and GLM, and concludes with GLM2IAC. Each sub-model is called in turn, processing CLM carbon information at the beginning of the sequence and eventually producing an updated land state that will be read by CESM throughout the model year (Fig. 4). The computational load of the IAC is dominated by GCAM and GLM, with the remaining subcomponents handling the processing needed to connect those models to each other and the rest of CESM. The IAC component includes the capability to read and write data between each step, thereby facilitating validation of each piece of code against corresponding offline versions and enabling detailed debugging for any differences revealed by the validation process. This validation and diagnostic capability has been implemented using NetCDF files to ensure the data exchanges are both self-descriptive and machine independent. 


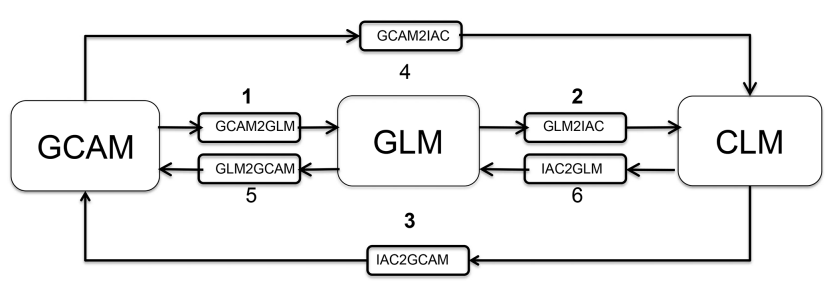

Figure 3. Schematic of the iESM interfaces among GCAM, GLM, and the CLM component of CESM. Several of these interfaces are unused in the initial implementation of the iESM.

\subsection{IAC2GCAM}

The IAC2GCAM interface translates and re-maps gridded information from CLM on its terrestrial carbon state into regional scaling factors (scalars) for crop yields and ecosystem carbon densities used by the agriculture and land use module internal to GCAM (Bond-Lamberty et al., 2014). The scalars represent our initial attempt to reconcile the separate carbon inventories either explicitly computed by or implicitly embedded via boundary data in the CLM, GCAM, GLM, and interface routines. In this initial version of iESM, the input to IAC2GCAM is read from CLM history files and includes the fields listed in Table 1 . The output consists of scalar fields for 27 crop and land cover fields on each of GCAM's 151 land units. The remapping between the CLM grid and GCAM regions is accomplished by translating CLM carbon, defined in broad terms of vegetative functional types, to the 27 specific GCAM crop/land types that lie at the heart of its economic, energy, and land use parameterizations. In addition to mapping between different land representations, IAC2GCAM also handles the temporal interpolation and spatial aggregation that is needed to represent CLM's gridded data in terms of the annually averaged regional values that GCAM requires. The spatial regridding process is aided by an external data file that specifies the areal overlap of CLM grid points with the GCAM land units. The mapping of CLM carbon to scalars applied to GCAM above and below-ground carbon is accomplished by averaging over the GCAM time step and then post-processing to remove outliers (Bond-Lamberty et al., 2014).

\subsection{GCAM}

The GCAM model produces worldwide land use projections incorporating information about demographics, economics, resources, energy production, and consumption (Sect. 3.2). Integration into iESM requires modifications to GCAM, including the addition of lightweight interface routines to CESM and the provision to share data in its XML database with these interface routines. In the current version of iESM, the input into GCAM consists of 27 crop and land cover scalars. The output from GCAM to the rest of the IAC component comprises the land surface areas for crop, pasture,
Table 1. Fields input by IAC2GCAM.

\begin{tabular}{ll}
\hline Variable & Definition \\
\hline cwdc & coarse woody debris carbon \\
totlitc & total litter carbon \\
totsomc & total soil organic matter carbon \\
deadrootc & non-respiring coarse root carbon \\
frootc & fine root carbon \\
livecrootc & respiring coarse root carbon \\
totvegc & total vegetation carbon \\
above- and below-ground carbon & \\
npp & net primary production \\
hr & heterotrophic respiration \\
\hline
\end{tabular}

forest, and the amount of harvested wood carbon for each of GCAM's 151 land units.

\subsection{GCAM2GLM}

The GCAM2GLM interface serves to allocate GCAM output from 151 land units to the $0.5^{\circ} \mathrm{GLM}$ grid. In the process, it also harmonizes the GCAM output to provide a smooth transition from historical land use data to future projections. The harmonization and regridding algorithms are based upon GLM historical simulations and the 2005 HYDE 3.0 historical land use data set (Klein Goldewijk et al., 2011). The inputs into the interface are the projections of crop, pasture, and forest area, as well as the amount of harvested wood carbon for 151 GCAM land units at the 5-year GCAM time step. The outputs from the interface are the areal extents of cropland, pasture, and forest at annual time steps on the GLM half-degree grid, together with a preprocessed version of the wood-harvest data readied for spatial allocation within GLM. The GCAM2GLM processing is contingent on the climate-change scenario under consideration and has embedded priorities for how the fractional areas of crop, pasture, and forested land are allocated. For example, these priorities could dictate that agricultural expansion happens preferentially on forested lands. A mechanism for recording and readily altering these embedded allocation priorities should be included in future versions of iESM.

\subsection{GLM}

In terms of its interactions with the rest of the current IAC components, the GLM model converts the annualized fractional land use states output by GCAM2GLM into gridded data sets suitable for input into CLM, while also computing the spatial pattern of wood-harvest area and the area of natural vegetation occupied by both primary and secondary vegetation. GLM converts the GCAM2GLM output data into a variety of fields on its native half-degree grid, nine of which are currently utilized in iESM including five wood-harvest categories (Table 2). GLM also calculates gross land use/land cover transitions within each year, but these are not used by CESM. 

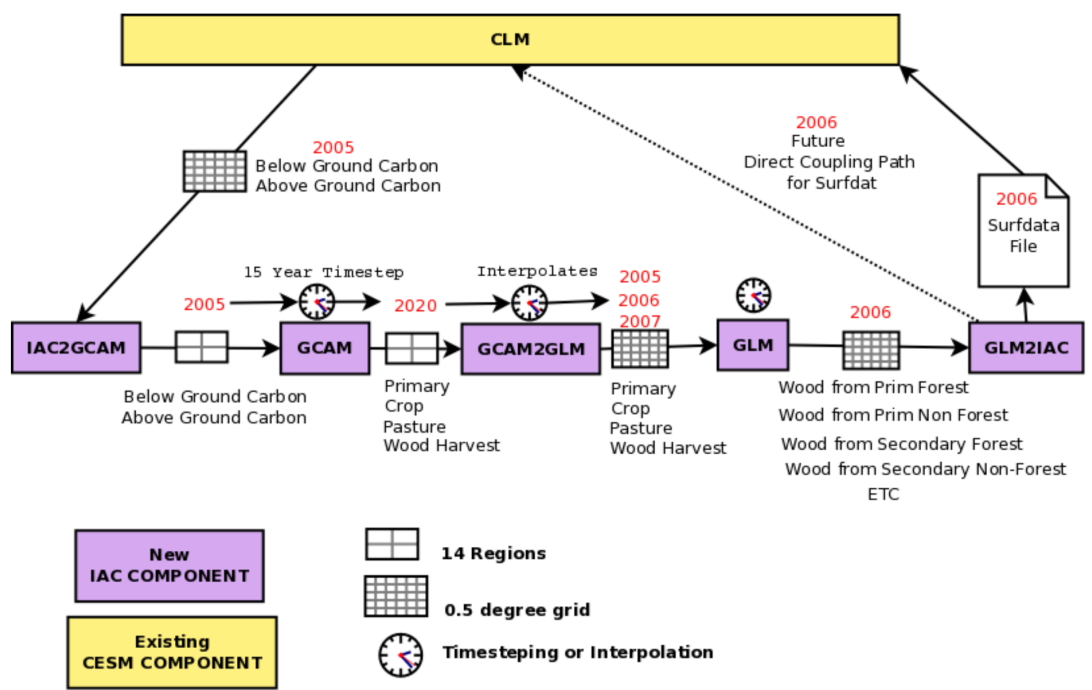

ETC

Figure 4. Sequence of operations and information exchanged during the time stepping of the iESM. Years are denoted in red, spatial interpolation with the grid patterns, time interpolation and time stepping with clocks, and model components with boxes.

Integration into CESM has required extensive modifications to GLM, including the redesign of data structures to reduce memory requirements and to accommodate control by CESM of its temporal evolution. Other modifications include the addition of restart functionality, the introduction of a control interface, the conversion of all boundary data into NetCDF, and the provision for routing all input and output through the calling interface.

\subsection{GLM2IAC}

The GLM2IAC interface is tasked with converting the harmonized outputs of GLM to time-varying data sets for land cover and wood-harvest area in CLM's native input format. The translation of GLM state and harvest variables to CLM land cover is based on code (Lawrence et al., 2012) to process the CMIP5 RCPs (Moss et al., 2010; van Vuuren et al., 2011), as well as on the external tool called mksurfdat (a contraction of "make surface data") used to generate CLM boundary data for the standard CESM. Both codes were inlined into the IAC component and are run interactively. The original land translation code has been extensively modified to better capture the afforestation signal generated in GCAM and has been renamed the LUT (Di Vittorio et al., 2014).

\subsection{CLM modifications}

Although CLM and the rest of CESM require minimal modifications to incorporate the IAC component, CLM was modified to permit updates to its time-varying input surface data sets after its initialization phase. This modification required introducing some changes in order to reread the time axis of the dynamic surface data set during the execution phase of CLM.

\subsection{Time stepping}

The IAC component advances in 1-year time steps and is called at the start of each calendar year. During this call, every sub-subcomponent in the IAC component is executed in order to prognose the time-varying CLM land surface data sets starting from the current CESM time step and ending 1 year into the future. To accomplish this, the IAC calculates the land surface for the time step 1 year in advance, then CLM interpolates between the current and future land surface at its native $30 \mathrm{~min}$ time step. In between the yearly IAC time steps, the IAC component is called monthly from CLM to create an annual average of CLM NPP (community land model net primary productivity) and HR (heterotrophic respiration) values. The GCAM subcomponent can be integrated using either one or three sequential 5-year time steps. The default is to use a 5-year time step and interpolate the yearly data needed for the rest of the IAC subcomponents. Prior to each GCAM call, the IAC computes the carbon scalars that constitute the feedback between CESM and GCAM.

\subsection{Technical issues}

Several technical requirements and protocols specific to large climate codes and CESM had to be introduced with the IAC component. The IAC component is bit-for-bit reproducible when rerun, and it restarts exactly from check-point files generated by previous runs. The IAC component is included in the CESM code repository and is tagged regularly in order to track code versions. A specific numerical experiment using the IAC in CESM can be described by specifying the model tag, the compset (which determines the model components), the grid, and a set of plain-text files that specify the 
Table 2. Fields output by GLM.

\begin{tabular}{ll}
\hline Variable & Definition \\
\hline gcrop & crop fraction \\
gpast & pasture fraction \\
gothr & primary land fraction \\
gsecd & secondary land fraction \\
gfvh1 & grid cell fraction that had wood harvested from primary forested land \\
gfvh2 & grid cell fraction that had wood harvested from primary non-forested land \\
gfsh1 & grid cell fraction that had wood harvested from mature secondary forested land \\
gfsh2 & grid cell fraction that had wood harvested from young secondary forested land \\
gfsh3 & grid cell fraction that had wood harvested from secondary non-forested land \\
\hline
\end{tabular}

features and input setting for the CESM components. The CESM configuration scripts have been augmented for iESM to include new compsets and new XML environment variables that specify items like the IAC mode (stub, data, active). The scripts have been further enhanced to incorporate several new libraries required by GCAM to support the opensource Berkeley DB XML (Oracle) database package with XQuery Access. These libraries include Berkeley DB XML, Berkeley DB, XQilla, and Xerces $\mathrm{C}++$, which must be installed before the active IAC component can be run within CESM.

To facilitate running the IAC with different CLM grids, many of the IAC settings are specified via namelist or read from files specified at run time. All the output data are written in NetCDF to ensure portability across computing platforms and to exploit the self-documenting features provided by this format. All variables are given explicit types, real variables are assigned to a type of double precision wherever possible, and the Fortran code complies with the CESM coding standard and is written in Fortran 90. Because the GLM and GCAM are written in $\mathrm{C}$ and $\mathrm{C}++$, Fortran- $\mathrm{C}$ interfaces have been implemented in several parts of the IAC component.

\section{Validation of the coupling among GCAM, GLM, and CESM}

One of the core requirements of the iESM design is to reproduce simulations conducted with the offline-coupled version of the same codes. Satisfaction of this requirement implies that the online-coupled simulations with iESM would be statistically indistinguishable from the offline-coupled simulations. Since the offline-coupled experiments have been configured to emulate the large number of simulations conducted using the same suite of codes for the CMIP5, successful reproduction of the offline-coupled runs would mean that the iESM user community could employ the large literature analyzing the CMIP5 runs to understand the baseline (or control) climatology and climate dynamics of iESM. Since iESM includes a variety of bug fixes and enhancements relative to the offline-coupled model configurations, the emulation will be only approximate.

The tests to verify the degree to which iESM reproduces the offline-coupled model have been conducted in three stages. First, with the exception of GLM, each component in iESM has been checked separately to show that, given the same input, the output of that component matches that of the corresponding component in the offline-coupled system to within the limits of machine precision (Sect. 6.1). In the case of GLM, there was extensive re-factoring of the code as well as conversion of many boundary data sets to NetCDF that resulted in differences that were greater than roundoff. Second, the development team has compared key climate properties from the iESM and offline-coupled system and has shown that differences between the two simulations of these properties are statistically indistinguishable from internal variability (Sect. 6.2). Third, the iESM team has validated the land use, land cover change, and CESM climate generated from the newer coupled iESM experiment using the CESM standard model diagnostics as well as specially constructed and quite comprehensive diagnostics for each of the components. Application of these diagnostics is covered in the papers describing the various iESM experiments and will not be repeated here (e.g., Jones et al., 2012, 2013).

\subsection{Verification of the interfaces among components}

These tests consist of comparing offline runs of each subcomponent of the offline-coupled implementation and online runs of iESM using the same forcing. To facilitate these tests, the iESM designers have allowed each of the components (GCAM, GLM, LUT, GCAM2GLM, etc.) to continue writing the state and diagnostic files that were output in the original offline models. Additionally, the data flowing between each of the component models were captured and written out in double precision NetCDF format. The ease of tracking the data flowing between each component as well as the ability of the component developers to continue using trusted analysis tools have allowed the iESM team to verify that the results produced by the offline and online versions of each subcom- 
ponent are, in general, identical to within the machine roundoff precision of the underlying calculations.

Once the individual pieces were validated, the team forced the IAC with prescribed CLM history output and compared the offline-coupled runs to the online runs with identical forcing. These simulations were designed to test that the feedbacks from the ESM to IA sub-systems of the IESM are as identical as possible between the offline-coupled and online versions. Both the offline-coupled and online IAC systems were subjected to the same external forcing from CLM, and the resulting dynamic surface data sets from both IAC versions were then compared. The team was able to verify that the results were identical to single-precision roundoff.

Finally, this test has been repeated with consistent and uniform time synchronization between CLM and IAC. Since the original test (described above) was forced with prescribed data, it did not ensure that the the temporal interactions between CLM and IAC were correctly reproduced in the online version relative to the implementation of the same interactions in the offline-coupled version. The team enhanced iESM to guarantee the same temporal interaction between CLM and the IAC in the two versions and also provided an alternative, reduced length GCAM time step of 5-year duration. The iESM also passed this more realistic test of its normal mode of operation, one in which there is cyclic twoway interaction between CLM and IAC coordinated by the master timing mechanism of the whole online model system.

\subsection{Comparison of climate states from uncoupled and coupled versions of iESM}

In order to test whether simulations from the offline-coupled and online iESM are statistically indistinguishable, we conducted a pair of integrations with these two versions of iESM based upon the RCP4.5 scenario. In these simulations, the copies of GCAM in both the offline-coupled and online versions are subjected to the same exogenous drivers and policy specifications that were used to create the original RCP4.5 scenario used in CMIP5. The two runs produce nearly identical future trajectories for global-mean surface air temperature. To formally evaluate this, we projected the time- and space-varying surface air temperature trajectories from these two simulations onto the spatial warming fingerprint (Santer et al., 2004) derived from the CCSM4 RCP4.5 CMIP5 ensemble mean, yielding a time series of projection coefficients for each simulation. We performed the same projection for each of six CCSM4 RCP4.5 ensemble members in order to quantify model internal variability with respect to this metric. Variation between the offline-coupled and online-coupled simulations, either in terms of the spatial pattern of warming or overall warming trend would cause these two trajectories to diverge. However, only $5.1 \%$ of the coefficients differed by more than the $95 \%$ confidence interval for unforced variability across the six-member ensemble of CCSM4 RCP4.5 simulations (Fig. 5). The unforced variability for the ensem-

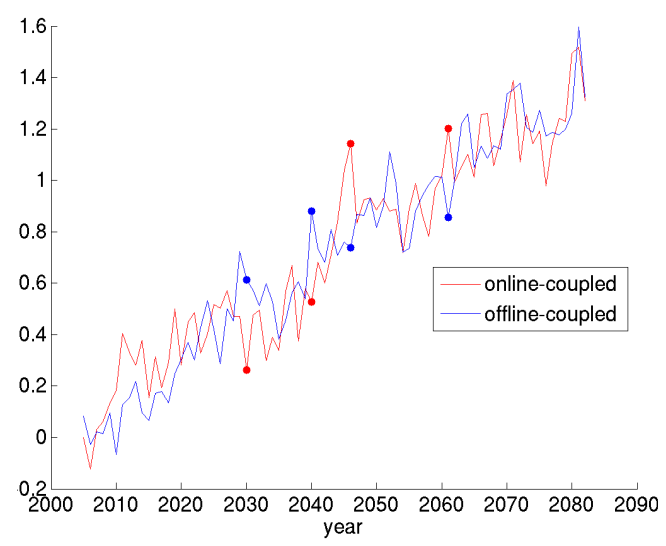

Figure 5. Projection coefficients for the online-coupled and offlinecoupled model implementations for a pair of equivalent scenarios based on RCP4.5. The coefficients are derived by projecting the spatial pattern of annual mean surface air temperature onto the "fingerprint" of the surface air warming trend derived from the RCP8.5 ensemble mean. The fingerprint is taken to be the first empirical orthogonal function of the 96-year time series of RCP8.5 annual mean surface temperatures, scaled so that its mean value is $1^{\circ} \mathrm{C}$. Circles indicate values that differ between the online-coupled and offlinecoupled simulations by more than the $95 \%$ confidence interval of this same metric calculated for the RCP4.5 6 member ensemble.

ble is generated by small perturbations to the date used to extract initial conditions from the end of a historical simulation terminating at the present day, and the resulting variability is manifested by different synoptic-scale weather but identical global climate across the ensemble. This test demonstrates that global-mean differences between the simulations from the offline-coupled and on-line versions of iESM are statistically indistinguishable from weather-related noise.

\section{Conclusions}

Several extensions to the iESM are already under development. First, capabilities have been developed for energysector components of the model to respond to climate change. These capabilities include developing the building sector so that demands for energy for heating and cooling are sensitive to temperature change (Zhou et al., 2013); developing thermoelectric plant sensitivity to ambient air temperature impacts on plant efficiency and water temperature impacts on plant operation; and developing model structure so that changes in climate (e.g., wind speed, solar irradiance) influence the supply curves of renewable energy sources (Zhou et al., 2012; Zhou and Smith, 2013). The development of these capabilities would be necessary for the eventual integration of climate information from CESM into the energysector operation of GCAM. Another area of development in iESM is the inclusion of supplies and demands for water, water management, and interactions of water resources with agriculture, the energy market, the hydrological cycle, and 
the rest of the climate system. New versions of GCAM fully track the water demands of energy and agriculture and incorporates a water-supply module that is sensitive to climate impacts (Hejazi et al., 2014, 2013). This major effort has positioned iESM to integrate water management and routing in subsequent phases of model development. Finally, the development of a new capability in GCAM to perform historical hindcast simulations (Chaturvedi et al., 2013) will enable the iESM model to be evaluated in terms of its ability to represent key land, water, and energy management decisions in response to historical driving conditions, as well as the climate implications of those decisions.

The first version of the iESM, however, already provides a significant new capability to the climate community. iESM represents the first coupled treatment of the human-climate system based on an IAM and ESM that both contributed to the most recent IPCC and US National Assessments and that support international communities of developers and investigators in integrated assessment and climate science. While iESM is designed to exploit the full capabilities of its parent models, it can be readily simplified and expanded due to its flexible and extensible architecture. The simplifications include inclusion or exclusion of human components, as well as potentially drastic reductions in the complexity and computational burden of the Earth system components by use of CESM's data modes. This capacity for faster execution helps ensure that iESM can be used to explore a large range of future scenarios of climate adaptation and mitigation in both a thorough yet economical manner. The possible expansions include inclusion of other IAMs that conform to the RCP handshake protocol, incorporation of additional forcing agents from the human system that can alter the climate system, and extension to simulate the supply and demand of other major resources, e.g., water, that interact strongly with natural and societal processes. This capacity for extensibility helps ensure that the iESM can and will continue to evolve with the state of integrated assessment and climate science.

\section{Code availability}

Copies of the code for the iESM version 1 are readily available upon request from the corresponding author.

Acknowledgements. This research was supported in part by the Director, Office of Science, Office of Biological and Environmental Research of the US Department of Energy under contract no. DE-AC02-05CH11231 to the Lawrence Berkeley National Laboratory as part of their Earth system modeling program. The authors used resources of the National Energy Research Scientific Computing Center (NERSC), also supported by the Office of Science of the US Department of Energy, under contract no. DE-AC02-05CH11231. The CESM project is supported by the National Science Foundation and the Office of Science (BER) of the US Department of Energy. Computing resources were provided by the Climate Simulation Laboratory at NCAR's Computational and Information Systems Laboratory (CISL), sponsored by the
National Science Foundation and other agencies. NCAR is sponsored by the National Science Foundation. The authors are also grateful for research support provided by the Integrated Assessment Research Program in the Office of Science of the US Department of Energy (DOE SC-IARP). This research used Evergreen computing resources at the Pacific Northwest National Laboratory's Joint Global Change Research Institute at the University of Maryland in College Park, which is supported by DOE SC-IARP. Pacific Northwest National Laboratory is operated by Battelle for the US Department of Energy under contract DE-AC05-76RL01830. The research was supported in part by support from the Office of Biological and Environmental Research of the US Department of Energy extended to the Oak Ridge National Laboratory. Oak Ridge National Laboratory is managed by UT-BATTELLE for the DOE under Contract DE-AC05-00OR22725. This research also used resources of the Oak Ridge Leadership Computing Facility at the Oak Ridge National Laboratory, which is supported by the Office of Science of the US Department of Energy under contract no. DE-AC05-00OR22725. The views and opinions expressed in this paper are those of the authors alone.

Edited by: G. A. Folberth

\section{References}

Bond-Lamberty, B., Calvin, K., Jones, A. D., Mao, J., Patel, P., Shi, X. Y., Thomson, A., Thornton, P., and Zhou, Y.: On linking an Earth system model to the equilibrium carbon representation of an economically optimizing land use model, Geosci. Model Dev., 7, 2545-2555, doi:10.5194/gmd-7-2545-2014, 2014.

Brovkin, V., Boysen, L., Arora, V. K., Boisier, J. P., Cadule, P., Chini, L., Claussen, M., Friedlingstein, P., Gayler, V., van den Hurk, B. J. J. M., Hurtt, G. C., Jones, C. D., Kato, E., de NobletDucoudré, N., Pacifico, F., Pongratz, J., and Weiss, M.: Effect of anthropogenic land-use and land-cover changes on climate and land carbon storage in CMIP5 projections for the twentyfirst century, J. Climate, 26, 6859-6881, doi:10.1175/JCLI-D-12$00623.1,2013$.

Calvin, K. V.: GCAM Wiki Documentation, available at: https:// wiki.umd.edu/gcam/ (last access: 21 August 2012), 2011.

CCSP: The Effects of Climate Change on Agriculture, Land Resources, Water Resources, and Biodiversity, a Report by the US Climate Change Science Program and the Subcommittee on Global Change Research, edited by: Backlund, P., Janetos, A., Schimel, D., Hatfield, J., Boote, K., Fay, P., Hahn, L., Izaurralde, C., Kimball, B. A., Mader, T., Morgan, J., Ort, D., Polley, W., Thomson, A., Wolfe, D., Ryan, M., Archer, S., Birdsey, R., Dahm, C., Heath, L., Hicke, J., Hollinger, D., Huxman, T., Okin, G., Oren, R., Randerson, J., Schlesinger, W., Lettenmaier, D., Major, D., Poff, L., Running, S., Hansen, L., Inouye, D., Kelly, B. P., Meyerson, L., Peterson, B., and Shaw, R., US Environmental Protection Agency, Washington, D.C., 362 pp., 2008.

Chaturvedi, V., Kim, S., Smith, S. J., Clarke, L., Yuyu, Z., Kyle, P., and Patel, P.: Model evaluation and hindcasting: An experiment with an integrated assessment model, Energy, 61, 479-490, doi:10.1016/j.energy.2013.08.061, 2013. 
Clarke, L., Edmonds, J., Krey, V., Richels, R., Rose, S., and Tavoni, M.: International climate policy architectures: overview of the EMF twenty-two international scenarios, Energ. Econ., 31, Supplement 2, S64-S81, doi:10.1016/j.eneco.2009.10.013, 2009.

Clarke, L. A., Edmonds, J. A., Jacoby, H. D., Pitcher, H. M., Reilly, J. M., and Richels, R. G.: Scenarios of greenhouse gas emissions and atmospheric concentrations, Tech. Rep. Subreport 2.1A of Synthesis and Assessment Product 2.1 by the US Climate Change Science Program and the Subcommittee on Global Change Research, Climate Change Science Program, Washington, D.C., 154 pp., 2007.

Community Earth System Model Project: available at: http://www2. cesm.ucar.edu/, last access: 14 July 2015.

Crook, J. A., Jones, L. A., Forster, P. M., and Crook, R.: Climate change impacts on future photovoltaic and concentrated solar power energy output, Energ. Environ. Sci., 4, 3101-3109, doi:10.1039/C1EE01495A, 2011.

de Lucena, A. F. P., Szklo, A. S., Schaeffer, R., de Souza, R. R., Borba, B. S. M. C., da Costa, I. V. L., Júnior, A. O. P., and da Cunha, S. H. F.: The vulnerability of renewable energy to climate change in Brazil, Energ. Policy, 37, 879-889, doi:10.1016/j.enpol.2008.10.029, 2009.

Di Vittorio, A. V., Chini, L. P., Bond-Lamberty, B., Mao, J., Shi, X., Truesdale, J., Craig, A., Calvin, K., Jones, A., Collins, W. D., Edmonds, J., Hurtt, G. C., Thornton, P., and Thomson, A.: From land use to land cover: restoring the afforestation signal in a coupled integrated assessment-earth system model and the implications for CMIP5 RCP simulations, Biogeosciences, 11, 6435-6450, doi:10.5194/bg-11-6435-2014, 2014.

Edmonds, J. and Reilly, J.: Global Energy: Assessing the Future, Oxford University Press, New York, 317 pp., 1985.

Feddema, J. J., Oleson, K. W., Bonan, G. B., Mearns, L. O., Buja, L. E., Meehl, G. A., and Washington, W. M.: The importance of land-cover change in simulating future climates, Science, 310, 1674-1678, doi:10.1126/science.1118160, 2005.

Foerster, H. and Lilliestam, J.: Modeling thermoelectric power generation in view of climate change, Reg. Environ. Change, 10, 327-338, doi:10.1007/s10113-009-0104-x, 2010.

Fung, I. Y., Doney, S. C., Lindsay, K., and John, J.: Evolution of carbon sinks in a changing climate, P. Natl. Acad. Sci. USA, 102, 11201-11206, doi:10.1073/pnas.0504949102, 2005.

GCRP: Global Climate Change Impacts in the United States: A State of Knowledge Report from the US Global Change Research Program, edited by: Karl, T. R., Melillo, J. M., and Peterson, T. C., Cambridge University Press, New York, 192 pp., 2009.

Golombek, R., Kittelsen, S. C., and Haddeland, I.: Climate change: impacts on electricity markets in Western Europe, Climatic Change, 113, 357-370, doi:10.1007/s10584-011-0348-6, 2012.

Hamududu, B. and Killingtveit, A.: Assessing climate change impacts on global hydropower, Energies, 5, 305-322, 2012.

Hejazi, M. I., Edmonds, J., Clarke, L., Kyle, P., Davies, E., Chaturvedi, V., Eom, J., Wise, M., Patel, P., and Calvin, K.: Integrated assessment of global water scarcity over the 21 st century - Part 2: Climate change mitigation policies, Hydrol. Earth Syst. Sci. Discuss., 10, 3383-3425, doi:10.5194/hessd-10-3383-2013, 2013.
Hejazi, M. I., Edmonds, J., Clarke, L., Kyle, P., Davies, E., Chaturvedi, V., Wise, M., Patel, P., Eom, J., and Calvin, K.: Integrated assessment of global water scarcity over the $21 \mathrm{st}$ century under multiple climate change mitigation policies, Hydrol. Earth Syst. Sci., 18, 2859-2883, doi:10.5194/hess-18-28592014, 2014.

Holt, E. and Wang, J.: Trends in wind speed at wind turbine height of $80 \mathrm{~m}$ over the contiguous United States using the North American Regional Reanalysis (NARR), J. Appl. Meteorol. Clim., 51, 2188-2202, doi:10.1175/JAMC-D-11-0205.1, 2012.

Hurrell, J. W., Holland, M. M., Gent, P. R., Ghan, S., Kay, J. E., Kushner, P. J., Lamarque, J. F., Large, W. G., Lawrence, D., Lindsay, K., Lipscomb, W. H., Long, M. C., Mahowald, N., Marsh, D. R., Neale, R. B., Rasch, P., Vavrus, S., Vertenstein, M., Bader, D., Collins, W. D., Hack, J. J., Kiehl, J., and Marshall, S.: The Community Earth System Model: a framework for collaborative research, B. Am. Meteorol. Soc., 94, 1339-1360, doi:10.1175/BAMS-D-12-00121.1, 2013.

Hurtt, G. C., Frolking, S., Fearon, M. G., Moore, B., Shevliakova, E., Malyshev, S., Pacala, S. W., and Houghton, R. A.: The underpinnings of land-use history: three centuries of global gridded land-use transitions, wood-harvest activity, and resulting secondary lands, Glob. Change Biol., 12, 1208-1229, doi:10.1111/j.1365-2486.2006.01150.x, 2006.

Hurtt, G. C., Chini, L. P., Frolking, S., Betts, R. A., Feddema, J., Fischer, G., Fisk, J. P., Hibbard, K., Houghton, R. A., Janetos, A., Jones, C. D., Kindermann, G., Kinoshita, T., Goldewijk, K. K., Riahi, K., Shevliakova, E., Smith, S., Stehfest, E., Thomson, A., Thornton, P., van Vuuren, D. P., and Wang, Y. P.: Harmonization of land-use scenarios for the period 1500-2100: 600 years of global gridded annual land-use transitions, wood harvest, and resulting secondary lands, Climatic Change, 109, 117-161, doi:10.1007/s10584-011-0153-2, 2011.

IPCC: Climate Change 2013: The Physical Science Basis. Contribution of Working Group I to the Fifth Assessment Report of the Intergovernmental Panel on Climate Change, edited by: Stocker, T. F., Qin, D., Plattner, G.-K., Tignor, M., Allen, S. K., Boschung, J., Nauels, A., Xia, Y., Bex, V., and Midgley, P. M., Cambridge University Press, Cambridge, United Kingdom and New York, NY, USA, 1535 pp., 2014.

Isaac, M. and van Vuuren, D. P.: Modeling global residential sector energy demand for heating and air conditioning in the context of climate change, Energ. Policy, 37, 507-521, doi:10.1016/j.enpol.2008.09.051, 2009.

Joint Global Change Research Institute: available at: http://www. globalchange.umd.edu/models/gcam/gcam-community/, last access: 14 July 2015.

Jones, A. D., Collins, W. D., Edmonds, J., Torn, M. S., Janetos, A., Calvin, K. V., Thomson, A., Chini, L. P., Mao, J., Shi, X., Thornton, P., Hurtt, G. C., and Wise, M.: Greenhouse gas policy influences climate via direct effects of land-use change, J. Climate, 26, 3657-3670, doi:10.1175/JCLI-D-12-00377.1, 2012.

Jones, A. D., Collins, W. D., and Torn, M. S.: On the additivity of radiative forcing between land use change and greenhouse gases, Geophys. Res. Lett., 40, 4036-4041, doi:10.1002/grl.50754, 2013.

Kim, S., Edmonds, J., Lurz, J., Smith, S., and Wise, M.: The ObjECTS framework for integrated assessment: hybrid modeling of transportation, Energ. J., 27, 63-91, 2006. 
Klein Goldewijk, K., Beusen, A., van Drecht, G., and de Vos, M.: The HYDE 3.1 spatially explicit database of human induced land use change over the past 12,000 years, Global Ecol. Biogeogr., 20, 73-86, 2011.

Kyle, G., Luckow, P., Calvin, K., Emanuel, W., Nathan, M., and Zhou, Y.: GCAM 3.0 agriculture and land use: data sources and methods, Tech. Rep. PNNL-21025, Pacific Northwest National Laboratory, 2011.

Labriet, M., Joshi, S., Vielle, M., Holden, P., Edwards, N., Kanudia, A., Loulou, R., and Babonneau, F.: Worldwide impacts of climate change on energy for heating and cooling, in: Mitigation and Adaptation Strategies for Global Change, 1-26, doi:10.1007/s11027-013-9522-7, Springer Netherlands, 2013.

Land-use Harmonization Project: available at: http://luh.umd.edu/, last access: 14 July 2015.

Lawrence, D. M., Oleson, K. W., Flanner, M. G., Thornton, P. E., Swenson, S. C., Lawrence, P. J., Zeng, X., Yang, Z.-L., Levis, S., Sakaguchi, K., Bonan, G. B., and Slater, A. G.: Parameterization improvements and functional and structural advances in Version 4 of the Community Land Model, Journal of Advances in Modeling Earth Systems, 3, M03001, doi:10.1029/2011MS000045, 2011.

Lawrence, P. J., Feddema, J. J., Bonan, G. B., Meehl, G. A., O'Neill, B. C., Oleson, K. W., Levis, S., Lawrence, D. M., Kluzek, E., Lindsay, K., and Thornton, P. E.: Simulating the biogeochemical and biogeophysical impacts of transient land cover change and wood harvest in the Community Climate System Model (CCSM4) from 1850 to 2100, J. Climate, 25, 3071-3095, doi:10.1175/JCLI-D-11-00256.1, 2012.

Linnerud, K., Mideksa, T. K., and Eskeland, G. S.: The impact of climate change on nuclear power supply, Energ. J., 32, 149-168, 2011.

Mao, J., Shi, X., Thornton, P. E., Piao, S., and Wang, X.: Causes of spring vegetation growth trends in the northern mid-high latitudes from 1982 to 2004, Environ. Res. Lett., 7, 014010, doi:10.1088/1748-9326/7/1/014010, 2012a.

Mao, J., Thornton, P. E., Shi, X., Zhao, M., and Post, W. M.: Remote sensing evaluation of CLM4 GPP for the period 2000-09, J. Climate, 25, 5327-5342, doi:10.1175/JCLI-D-11-00401.1, $2012 \mathrm{~b}$.

Mao, J., Shi, X., Thornton, P. E., Hoffman, F. M., Zhu, Z., and Myneni, R. B.: Global latitudinal-asymmetric vegetation growth trends and their driving mechanisms: 1982-2009, Remote Sensing, 5, 1484-1497, doi:10.3390/rs5031484, 2013.

Matthews, H., Weaver, A., Meissner, K., Gillett, N., and Eby, M.: Natural and anthropogenic climate change: incorporating historical land cover change, vegetation dynamics and the global carbon cycle, Clim. Dynam., 22, 461-479, doi:10.1007/s00382004-0392-2, 2004.

Moss, R. H., Edmonds, J. A., Hibbard, K. A., Manning, M. R., Rose, S. K., van Vuuren, D. P., Carter, T. R., Emori, S., Kainuma, M., Kram, T., Meehl, G. A., Mitchell, J. F. B., Nakicenovic, N., Riahi, K., Smith, S. J., Stouffer, R. J., Thomson, A. M., Weyant, J. P., and Wilbanks, T. J.: The next generation of scenarios for climate change research and assessment, Nature, 463, 747-756, doi:10.1038/nature08823, 2010.

Oleson, K., Lawrence, D., Bonan, G., Flanner, M., Kluzek, E., Lawrence, P., Levis, S., Swenson, S., and Thornton, P.: Technical description of version 4.0 of the Community Land Model
(CLM), Tech. Rep. NCAR/TN-478+STR, National Center for Atmospheric Research, 2010.

Oleson, K., Lawrence, D., Bonan, G., Drewniak, B., Huang, M., Koven, C., Levis, S., Li, F., Riley, W., Subin, Z., Swenson, S., Thornton, P., Bozbiyik, A., Fisher, R., Heald, C., Kluzek, E., Lamarque, J.-F., Lawrence, P., Leung, L., Lipscomb, W., Muszala, S., Ricciuto, D., Sacks, W., Sun, Y., Tang, J., and Yang, Z.-L.: Technical description of version 4.5 of the Community Land Model (CLM), Tech. Rep. NCAR/TN503+STR, National Center for Atmospheric Research, 2013.

Pielke, R. A., Marland, G., Betts, R. A., Chase, T. N., Eastman, J. L., Niles, J. O., Niyogi, D. D. S., and Running, S. W.: The influence of land-use change and landscape dynamics on the climate system: relevance to climate-change policy beyond the radiative effect of greenhouse gases, Philos. T. Roy. Soc. A, 360, 17051719, 2002.

Pitman, A., de Noblet-Ducoudré, N., Cruz, F., Davin, E., Bonan, G., Brovkin, V., Claussen, M., Delire, C., Ganzeveld, L., Gayler, V., van den Hurk, B., Lawrence, P., van der Molen, M., Müller, C., Reick, C., Seneviratne, S., Strengers, B., and Voldoire, A.: Uncertainties in climate responses to past land cover change: first results from the LUCID intercomparison study, Geophys. Res. Lett., 36, L14814, doi:10.1029/2009g1039076, 2009.

Ruebbelke, D. and Voegele, S.: Impacts of climate change on European critical infrastructures: the case of the power sector, Environ. Sci. Policy, 14, 53-63, doi:10.1016/j.envsci.2010.10.007, 2011.

Ruebbelke, D. and Voegele, S.: Short-term distributional consequences of climate change impacts on the power sector: who gains and who loses?, Climatic Change, 116, 191-206, doi:10.1007/s10584-012-0498-1, 2013.

Santer, B., Wigley, T., Simmons, A., Kallberg, P., Kelly, G., Uppala, S., Ammann, C., Boyle, J., Bruggemann, W., Doutriaux, C., Fiorino, M., Mears, C., Meehl, G., Sausen, R., Taylor, K., Washington, W., Wehner, M., and Wentz, F.: Identification of anthropogenic climate change using a second-generation reanalysis, J. Geophys. Res., 109, D21104, doi:10.1029/2004JD005075, 2004.

Schaeffer, M., Eickhout, B., Hoogwijk, M., Strengers, B., van Vuuren, D., Leemans, R., and Opsteegh, T.: $\mathrm{CO}_{2}$ and albedo climate impacts of extratropical carbon and biomass plantations, Global Biogeochem. Cy., 20, GB2020, doi:10.1029/2005GB002581, 2006.

Shi, X., Mao, J., Thornton, P. E., Hoffman, F. M., and Post, W. M.: The impact of climate, $\mathrm{CO}_{2}$, nitrogen deposition and land use change on simulated contemporary global river flow, Geophys. Res. Lett., 38, L08704, doi:10.1029/2011GL046773, 2011.

Shi, X., Mao, J., Thornton, P. E., and Huang, M.: Spatiotemporal patterns of evapotranspiration in response to multiple environmental factors simulated by the Community Land Model, Environ. Res. Lett., 8, 024012, doi:10.1088/1748-9326/8/2/024012, 2013.

Snyder, P., Delire, C., and Foley, J.: Evaluating the influence of different vegetation biomes on the global climate, Clim. Dynam., 23, 279-302, doi:10.1007/s00382-004-0430-0, 2004.

Taylor, K. E., Stouffer, R. J., and Meehl, G. A.: An overview of CMIP5 and the experiment design, B. Am. Meteorol. Soc., 93, 485-498, doi:10.1175/BAMS-D-11-00094.1, 2011.

Thomson, A. M., Calvin, K. V., Smith, S. J., Kyle, G. P., Volke, A., Patel, P., Delgado-Arias, S., Bond-Lamberty, B., Wise, M. A., 
Clarke, L. E., and Edmonds, J. A.: RCP4.5: a pathway for stabilization of radiative forcing by 2100 , Climatic Change, 109 , 77-94, doi:10.1007/s10584-011-0151-4, 2011.

University of Maryland Global Ecology Lab: available at: http://gel. umd.edu/glm.php, last access: 14 July 2015.

van Ruijven, B. J., van Vuuren, D. P., de Vries, B. J. M., Isaac, M., van der Sluijs, J. P., Lucas, P. L., and Balachandra, P.: Model projections for household energy use in India, Energ. Policy, 39, 7747-7761, doi:10.1016/j.enpol.2011.09.021, 2011.

van Vliet, M. T. H., Yearsley, J. R., Ludwig, F., Voegele, S., Lettenmaier, D. P., and Kabat, P.: Vulnerability of US and European electricity supply to climate change, Nature Climate Change, 2, 676-681, doi:10.1038/NCLIMATE1546, 2012.

van Vuuren, D. P., den Elzen, M. J., Lucas, P., Eickhout, B., Strengers, B., van Ruijven, B., Wonink, S., and van Houdt, R.: Stabilizing greenhouse gas concentrations at low levels: an assessment of reduction strategies and costs, Climatic Change, 81, 119-159, doi:10.1007/s10584-006-9172-9, 2007.

van Vuuren, D. P., Edmonds, J., Kainuma, M., Riahi, K., and Weyant, J.: A special issue on the RCPs, Climatic Change, 109, 1-4, doi:10.1007/s10584-011-0157-y, 2011.

van Vuuren, D. P., Bayer, L. B., Chuwah, C., Ganzeveld, L., Hazeleger, W., van den Hurk, B., van Noije, T., O’Neill, B., and Strengers, B. J.: A comprehensive view on climate change: coupling of earth system and integrated assessment models, Environ. Res. Lett., 7, 024012, doi:10.1088/1748-9326/7/2/024012, 2012.

Voldoire, A., Eickhout, B., Schaeffer, M., Royer, J.-F., and Chauvin, F.: Climate simulation of the twenty-first century with interactive land-use changes, Clim. Dynam., 29, 177-193, doi:10.1007/s00382-007-0228-y, 2007.

Wise, M. and Calvin, K.: GCAM 3.0 agriculture and land use: technical description of modeling approach, Tech. Rep. PNNL-20971, Pacific Northwest National Laboratory, available at: https://wiki.umd.edu/gcam/images/ 8/87/GCAM3AGTechDescript12_5_11.pdf (last access: 14 July 2015), 2011.
Wise, M., Calvin, K., Kyle, G., Luckow, P., and Edmonds, J.: Economic and physical modeling of land use in GCAM 3.0 and an application to agricultural productivity, land, and terrestrial carbon, Climate Change Economics, 5, 1450003, doi:10.1142/S2010007814500031, 2014.

Yu, S., Eom, J., Zhou, Y., Evans, M., and Clarke, L.: Scenarios of building energy demand for China with a detailed regional representation, Energy, 67, 284-297, doi:10.1016/j.energy.2013.12.072, 2014.

Zhang, Y., Smith, S. J., Kyle, G. P., and Stackhouse Jr., P. W.: Modeling the potential for thermal concentrating solar power technologies, Energ. Policy, 38, 7884-7897, doi:10.1016/j.enpol.2010.09.008, 2010.

Zhou, Y. and Smith, S. J.: Spatial and temporal patterns of global onshore wind speed distribution, Environ. Res. Lett., 8, 034029, doi:10.1088/1748-9326/8/3/034029, 2013.

Zhou, Y., Luckow, P., Smith, S. J., and Clarke, L.: Evaluation of global onshore wind energy potential and generation costs, Environ. Sci. Technol., 46, 7857-7864, doi:10.1021/es204706m, 2012.

Zhou, Y., Eom, J., and Clarke, L.: The effect of global climate change, population distribution, and climate mitigation on building energy use in the US and China, Climatic Change, 119, 979 992, doi:10.1007/s10584-013-0772-x, 2013.

Zhou, Y., Clarke, L., Eom, J., Kyle, P., Patel, P., Kim, S. H., Dirks, J., Jensen, E., Liu, Y., Rice, J., Schmidt, L., and Seiple, T.: Modeling the effect of climate change on U. S. state-level buildings energy demands in an integrated assessment framework, Appl. Energ., 113, 1077-1088, doi:10.1016/j.apenergy.2013.08.034, 2014. 